\title{
Google Map Based User Interface for Network Resource Planning in Post Disaster Management
}

\author{
Sujoy Saha \\ National Institute of \\ Technology,Durgapur,India \\ sujoy.ju \\ @gmail.com
}

\author{
Nitin Agarwal \\ Institute of engineering \& \\ Management,Kolkata,India \\ nitin jdbi \\ @rediffmail.com
}

\author{
Subrata Nandi \\ National Institute of \\ Technology,Durgapur,India \\ subrata.nandi \\ @cse.nitdgp.ac.in
}

\begin{abstract}
When disaster strikes, existing network infrastructure is jeopardized. Network Resource Planning is required for building a cost-effective communication system, to fill the communication gap. Ensuring higher packet delivery ratio along with optimal utilization of resources are prime objectives of such planning. We have designed a Web-based application based on the four tier planned hybrid architecture which conforms the aforesaid motives yielding a desired performance, for a given disaster hit area map using suitable heuristic algorithms.
\end{abstract}

\section{Keywords}

Post Disaster Management, Network Resource Planning, Google Map API, Web Application

\section{INTRODUCTION}

In 2011, Japan took control over devastating tsunami in just ten days by using sophisticated technical support. Satellite phones and emergency-use portable communication facilities were supplied by Japan's Ministry of Internal Affairs at no cost. Unmanned Aircrafts were used to assess infrastructural damage. Also, robots were sent around 2,500 metres to the seabed to repair damaged cables. Following Japan for any developing country, like India, would be challenging in terms of cost and planning. Hence developing a cost effective system due to varying socio-economic conditions is the chief motive for undertaking this research. Consequently we have developed a customized Google map based User Interface, based on four tier hybrid architecture[1] as shown in figure 1, where disaster Affected Area(AA) is divided into Shelter Points(SP). Within each SP, DTN Nodes[Tier-1] are utilized to deliver packets periodically to the nearest DropBox[Tier-2] belonging to each SP. As the DTNs formed are sparse, and DBs are far apart, vehicles used by rescue/relief teams, equipped with Wi-Fi and VSAT (for emergency messages), can act as Data Mules (DMs)(mechanical backhauls) [Tier-3] to carry informations within desired Latency. DBs are grouped by using an efficient clustering algorithm and at each group center one (NLOS/near LOS) Wi-Fi tower (WTs) [Tier-4], accumulating data from a nonoverlapping set of DBs will be placed. In above mentioned planned[2] architecture, two objectives need to be met, given the geographic and geodesic distance between each DropBox: Objective 1: Finding the optimal number of groups along with the placement of Wi-Fi tower on each Group Center. Objective 2: Finding the minimum number of DMs

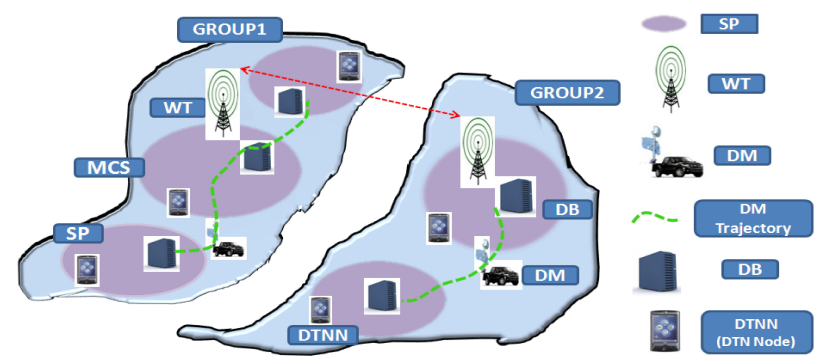

Figure 1: Four Tier hybrid Architecture using DTN Nodes, DropBoxes(DBs), DataMules(DMs) and WiFi Towers(WTs)

required for each group to traverse those within L \& finding the pathways of each DM.

We have implemented heuristic algorithm for meeting each of the above objective using Google Maps API.

A customized user-interface is developed to show that the disaster hit area can be marked on a dynamic Google map following its subdivision into SPs with DBs and strategic placements of Wi-Fi towers along with the DM pathways based on the aforesaid architecture under latency constraint ensuring optimal utilization of resources.

\section{GOOGLE MAP API}

Google Maps has a wide array of APIs that lets us embed the robust functionality and everyday usefulness of Google Maps into our own website and applications, and overlay our own data on top of them. Among the multitude APIs, Google Maps JavaScript API v3.9 [3] is used that lets us embed dynamic map into a web page and provides a number of utilities for manipulating maps.

\section{GOOGLE MAP BASED WEB USER IN- TERFACE}

In the Web User Interface(UI) along with the integration of google map, various Web elements are also placed around the map, manipulating it dynamically to produce an effective Post Disaster Management plan based on the four tier hybrid architecture. The Post Disaster Relief team can find out the Affected Area(AA) on the map by furnishing the location along with the extent of the area approximately affected by the disaster. The location entered here is sent to the Google server for geocoding[4]. The approximate range of the area can be set on the map using any one of the option 
namely 100-200 Sq.km or 200-500 Sq.km. Once the area is set on the UI a rectangular region is created on the map to highlight the AA.

Inside the AA a user can directly right click on the map to create a closed graph until the required number of edges are formed. This closed graph is viewed as Shelter Point(SP). The geodesic area of the resultant SP is recorded in the UI for the sake of further modification. Likewise, a number of SPs can be created. Thereafter DropBoxes can be created on the Map at any point with in a SP or closed graph using drawing tool, placed at the Top Center on the Map.

\section{IMPLEMENTATION OF HEURISTIC SO- LUTIONS}

Once the SPs and DBs are positioned on the Google map, heuristic algorithms can be executed upon. Input to this algorithm is made dynamic allowing user to set values as per disaster conditions. We, for sample sake, have taken Allowable Time(AT) to be 120 minutes assuming 5 minutes is required for covering a $\mathrm{km}$. Service Time(ST) consumed by Data Mule for uploading and downloading data to and from $\mathrm{DB}$ is set to 25 minutes and the $\mathrm{Wi}-\mathrm{Fi}$ tower range is considered to be $10 \mathrm{~km}$. Here $12 \mathrm{DBs}$ are positioned into corresponding SPs.

At the outset geodesic and geographical distance of DBs

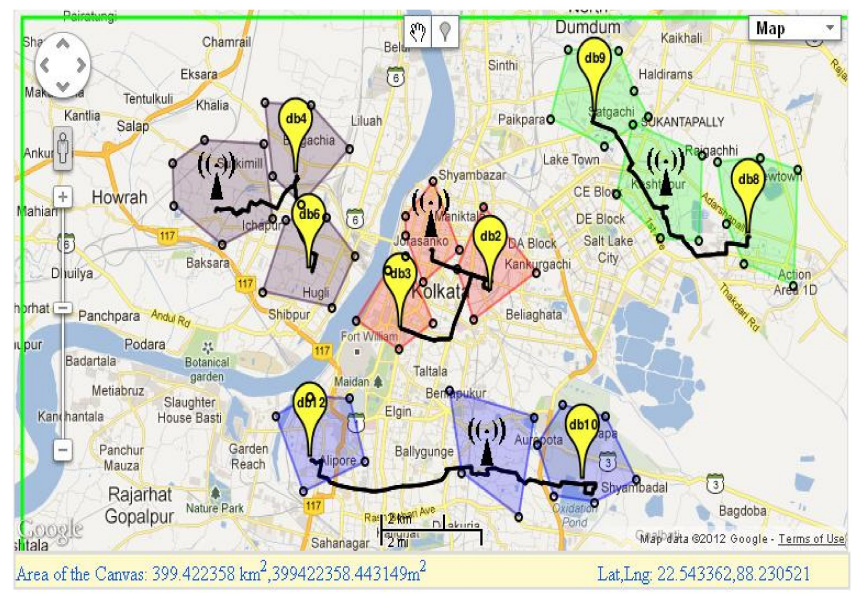

Figure 2: Four Groups are formed with Wi-Fi Tower placed on Group Center along with the Data Mule (DMs) trajectory

in all permutation is obtained using Google map API. Subsequently the set of distance is reduced to include only the combination having shortest distance. While the Distance between dropboxes are obtained, the conditions of pathways is also taken into account. If a path is blocked on account of disaster, it is reflected on the google map in the form of red color path. It returns a prompt stating whether the path is blocked or not i.e. whether traversal between Dropboxes is possible or not using the existing pathway. Distance obtained is utilized to divide the Activity Area into various groups. Each group has atleast one SP which is Group Center and can have zero or more SPs as group members depending on the proximity of the SPs with the group center as per the heuristic algorithm. Among all the groups one group center is selected as Master Control Station(MCS) which might have connectivity beyond the AA or with post disaster relief team. Group formation is shown in figure 2 where SPs are colored as per their group and Wi-Fi tower is placed on each group center.

For facilitating information flow from group members to group center DMs are deployed. A DM traverses from group center to a group member and continues traversing to other group members, as shown in figure 2, subject to latency which is sum total of ST(for each SP), geographical distance between group center and DBs including return distance. In figure 3 it is shown that Group 1 requires 1 DM which traverses from DB1 to DB2 and then from DB2 to DB3 and subsequenty returns from DB3 to DB1 with in acceptable latency. A group can have one or more DM as shown in figure 3. Once data corresponding to each $\mathrm{SP}$ is received by respective group center, it is sent to the MCS using Wi-Fi towers. A MCS would subsequently send it to the Relief team for the sake of rescuing victims and bringing forth relief aid.

\begin{tabular}{|c|c|}
\hline Group Number & Data Mule \\
\hline Group 1 & \multirow{2}{*}{ No of DM: 1} \\
\hline & \\
\hline $\begin{array}{l}\text { Group Center: }{ }^{D B} 1 \\
\text { Group Members: } \mathrm{DB}^{2} 2, \mathrm{DB} 3\end{array}$ & $\mathrm{DB} 1 \rightarrow \mathrm{DB} 2 \rightarrow \mathrm{DB} 3$ \\
\hline Group 2 & No of DM: 2 \\
\hline $\begin{array}{l}\text { Sroup center: } D B \quad 7 \\
\text { Group Members: DB } 8, D B \quad 9\end{array}$ & $\begin{array}{l}\mathrm{DB} 7 \rightarrow>\text { DB } 9 \\
\text { DB7 } \rightarrow>\text { DB8 }\end{array}$ \\
\hline Group 3 & No of DM: 2 \\
\hline $\begin{array}{l}\text { Center: DB } 11 \\
\text { Members: } D B \text {, } 10, D B\end{array}$ & $\begin{array}{l}\text { DB11 }>\text { DB10 } \\
\text { DB11->DB12 }\end{array}$ \\
\hline Group 4 & No of DM: 1 \\
\hline $\begin{array}{l}\text { Group center: DB } 5 \\
\text { Group Members: }{ }^{D B} 4, \mathrm{DB} 6\end{array}$ & $\mathrm{DB} 5 \rightarrow \mathrm{DB} 4 \rightarrow \mathrm{DB} 6$ \\
\hline
\end{tabular}

Figure 3: Group Details and their corresponding Data Mules(DMs)

\section{CONCLUSION}

The design of UI has been kept clear and concise for the sake of its convenient deployment to create efficient post disaster management plan. Using this UI Rescue team can rapidly determine the optimal resources required for setting up communication system ensuring higher packet delivery ratio within acceptable latency. Presently we are working on the following: (1)Incorporating altitude of a region to trace the water level during Disaster;(2)Development of Android application for accumulating information from the disaster Affected Area.

\section{REFERENCES}

[1] S.Saha, V.K. Shah, R. Verma, R. Mandal and S.Nandi, Is It Worth Taking a Planned Approach to Design Ad-hoc Infrastructure for Post Disaster Communication?, in Proceedings of the ACM CHANTS'12, co-located with MobiCom 2012, August 22, 2012, Istanbul, Turkey.

[2] https://sites.google.com/site/dtnproject/

[3] Google Maps Javasript API V3 Reference::https://developers.google.com/maps/ documentation/javascript/reference.

[4] Geocoding Service::https://developers.google.com /maps/documentation/javascript/geocoding. 